\title{
INDUSTRIAL CARBON AND GRAPHITE
}

$\mathrm{T}$ HE rapidly developing interest in carbon and graphite was demonstrated by the presentation of more than sixty papers, running in preprint form to more than 500 pages, to the Conference on Industrial Carbon and Graphite organized by the Society of Chemical Industry and held in London during September 24-26. In part, the enhanced importance of these materials has arisen from the employment of graphite as a moderator in nuclear-power production, but the use of industrial carbon in many other branches of industry has also increased greatly in the past two decades.

The Conference was opened by Dr. H. K. Cameron, who surveyed the traditional uses of carbon, and modern developments from them, and ventured to predict some of the problems of carbon technology which might be solved in the future.

Historically, the expanding industrial use of carbon has depended mainly upon the evolution of a technology for the conversion of the irregular piece of coke or char resulting from the carbonization of organic matter into strong, rigid, macroscopically homogeneous forms suitable for industrial application. The basic process has remained unchanged for a century. The primary coke is ground (to form the 'grist'), mixed with a suitable binder (such as tar or pitch), pressed or extruded into the desired shape and calcined. The binder decomposes to form a secondary coke which links together the particles of the primary coke to produce a rigid product. At this stage the material is called 'industrial carbon'. In recent years a further process is often added in which the carbon is heated in an inert atmosphere (such as argon) to temperatures in excess of $2,000^{\circ} \mathrm{C}$. to induce graphitization. The product is "industrial graphite'.

Materials prepared in this way find many applications. Among those dealt with specifically at the Conference were: as electrodes in both electrochemical furnaces (used, for example, in aluminium production) and in melting furnaces; as moulds, crucibles and dies in metallurgical processes ; in the construction of chemical plant to withstand either high temperatures or corrosive conditions ; as carbon brushes for electrical machines; as dry bearings; as electrical resistors both in the electronics industry and in heavier electrical applications; and finally as a moderator in nuclear piles.

Industrial carbon and graphite differ from natural graphite in many important respects, most of which were referred to during the Conference. These differences are structural rather than chemical in origin, and are associated with features ranging from variations in the erystal lattice of sub-microscopic regions of the material, to heterogeneity on the microscopic and occasionally on the macroscopic scale which results inevitably from the method of manufacture. The properties of the final product depend on many factors, including the nature of the basic raw material, the way in which it is carbonized, the method of grinding, the size distribution and the shape of the grist particles, the nature of the binding agent and the amount used, the method of forming the product (pressing or extrusion), the conditions of the second carbonization and finally the temperature of graphitization and the atmosphere in which it is carried out. These factors often interact with one another, so that the problem of understanding the nature and properties of a given industrial carbon or graphite in relation to its method of preparation is exceedingly complex, and in the absence of a fundamental understanding, a substantial body of empirical technique has been built up in the industry. The papers presented to the Conference show, however, that real attempts are being made to rationalize knowledge of the fundamental processes, and to relate this knowledge to the properties of the final product by the correlation of information derived from a wide range of experimental methods.

The property which reflects most directly the difference between artificial carbons and natural graphite is the bulk density : industrial materials rarely exceed a density of $1.75 \mathrm{gm} . / \mathrm{cm} .^{3}$, whereas graphite has a density of $2 \cdot 26 \mathrm{gm} . / \mathrm{cm} .^{3}$. This difference arises from structural differences at all size-levels. It is generally presumed (although not definitely proved in all cases) that the basic structural unit of carbonized materials is the $\mathrm{C}_{b}$-ring, although the presence of some side-chains with free valencies is also assumed. Evidence for the presence of free radicals comes from electron-resonance studies (Ingram). These rings are fused together to form planar aggregates which may be complete or possess 'holes' of various kinds. X-ray studies show that natural graphite consists of perfect planes of fused rings, arranged parallel to one another with a definite interlayer spacing, and with the orientation of each plane relative to its neighbour completely defined. The energies of the electronic configurations in the graphite structure are not yet calculable with high accuracy, and an attempt to obtain evidence about them by studying the reflectance of light of various wave-lengths by polished graphites was reported to the present Conference by Humphreys-Owen and Gilbert. Less-perfect structures of lower density occur when the relative orientation of the rings in adjacent planes is random, and the average distance between planes increases slightly: the structure is said to be 'turbostratic'. Amorphous carbons have also been subjected to careful study by X-ray techniques, and it is concluded that they consist of graphite-like crystallites, the size of which may range from the order of ten angstrom units to several hundred, interspersed with turbostratic and possibly even more randomly arranged carbon. In addition, nearly all carbons have a characteristic porous structure consisting of cavities the dimensions of which may range from a few angstroms to tenths of a millimetre, and the shapes of which may vary widely. Each individual carbonized material has its own characteristic structure, while the industrial carbons under discussion consist of an intimate blending of two such structures, one arising from the primary coke and the other from decomposition of the binder. Some of the more important implications of this two-component structure were brought out in Mrozowski's general introduction to the Conference, which stressed not only the complexities of the microstructure of artificial carbons, but also 
the analogy between them and compressed powders.

X-ray studies have formed the basis of many of the conclusions regarding the molecular structure of carbon and graphite, and a survey of diffraction methods (including neutron diffraction) was given by Bacon, while a rapid X-ray method for determining the preferred crystallographic orientation was described by Ali, Fitzer and Ragoss. Recent work on the structure of carbonized coals was reported by Diamond and Hirsch, while the carbonization products of materials of known composition have been examined by Stadler and Thomson (single crystals of flavanthrone) and by Winslow, Matreyek and Yager (vinyl polymers). Further work on the progress of graphitization at high temperatures, as revealed by X-ray studies, was reported by Maire and Mering, and by Steward and H. W. Davidson. Correlation is found by Adamson and Blayden between the size of the graphite crystals and the magnetic suscept. ibility, and these authors used the latter property to study the dependence of crystallite size on starting material and thermal treatment. Other properties which are closely related to the structure of carbons are the thermal and electrical conductivities. They depend mainly on the size, orientation and density of the crystallites, but in different ways in various ranges of temperature. Consequently, measurements of thermal and electrical conductivity over a wide temperature-range are important (Berman ; Powell ; Albert and Parisot; Mason), especially when associated with X-ray studies (Bowman, Krumhansl and Meers). The porosity of carbons is a property which in some applications is desirable and for many others undesirable. Reliable methods for assessing the total porosity and its distribution in terms of the size of cavities are therefore important. Bond and Spencer reviewed methods developed at the British Coal Utilization Research Association for studying the ultra-fine structure of coals and carbonized coals, while Academician Dubinin surveyed Russian work on the correlation of adsorptive properties with porous structure. A paper by Cadenhead and Everett was concerned with the elucidation of the porous structure of carbons by a study of the thermodynamics of adsorption processes. Gas permeability methods are widely used for assessing porosity, although the fundamental theory of the method is far from satisfactory. Papers by Wiggs and by Hutcheon, Longstaff and Warner discussed both the theory and the interpretation of experimental results on industrial graphites.

The major part of the porosity of an industrial carbon is associated with the secondary coke formed by decomposition of the binder. Consequently, it is desirable to use the minimum amount of binder. The grist aggregate must therefore have a minimum of inter-particle voids, and optimum packing is achieved by blending different particle sizes in the grist. The shape as well as the size of grist particles is also important, and this has been studied by Amstein and Watson. Furthermore, extruded carbons are anisotropic and this is in part controlled by the particle shape (Grossman). The choice of suitable binder is of great importance, and several papers (Darney; MeNeil and Wood; Blakeley and Earp ; Lossing) were devoted to this problem. A systematic investigation of the variation of the properties of carbons, in particular mechanical strength and elec. trical resistivity, with amount of binder and with the composition of the grist, was reported by Fialkov.
The manufacture of electrodes having long life is important economically, both in the production of aluminium and in electro-melting: half a ton of carbon is consumed for each ton of aluminium produced, while 15 per cent of the cost of converting scrap steel to ingots arises from electrode wear. Two problems in the production of electrodes for aluminium cells were discussed. The first is concerned with the cracking of electrodes during baking, which causes uneven current distribution. According to Pratt, this arises from shrinkage of the grist during the second carbonization, and he has studied the shrinkage of cokes at $1,000^{\circ} \mathrm{C}$.; this is shown to be related to the hydrogen content of the coke. Disintegration of the electrode surface, arising from preferential oxidation of one component of the carbon, also makes an important contribution to electrode wear, and Bowitz, Böckman, Jahr and Sandberg attribute this to the greater porosity and reactivity of the coked binder. The mechanism of electrode consumption in melting furnaces is not clearly understood, and the work of Ravenscroft and Preston on the distribution of electrode wear at different points on the surface as a function of electrode current and voltage provides some basic information about the phenomenon.

Problems of electrode life, as well as many industrial applications of carbon, depend upon the reactivity of carbons towards oxidizing gases. A fundamental study by J. D. Watt and Franklin in which changes of structure arising from oxidation were studied by $\mathrm{X}$-ray methods suggests that the most reactive part of a porous carbon structure consists of single, unstacked, graphite layers which can adsorb oxygen on both faces. The kinetics of reaction of cerbon with various gases continues to be studied, and a number of recent researches were reported (Graham, A. R. G. Brown, Hall and W. Watt ; Earp and Hill; Walker and Nichols; Day, Walker and Wright); particular emphasis is now being placed on measurements at high temperatures $\left(1,500-2,500^{\circ}\right.$ C.) and at high rates of gas flow. The effect of surface oxides on the reactivity of carbon was the subject of a paper by Harker, Marsh and WynneJones.

In the electrical industry great importance is attached both to the electrical conductivity of carbon, and to the contact resistance at carbon-carbon and carbon-metal interfaces. A review of developments in applications of carbon to current-collection was given by Vaughan, with particular reference to behaviour under high-altitude conditions, where, in the absence of water vapour, the friction between brush and metal commutator increases and rapid wear ensues. Experimental studies of the electrical properties of sliding contacts at low gas-pressures were described by Millet. In these applications metallized types of graphite are often employed, and details of the production of copper-graphite materials were given by Beech and Price. The contact resistance at carboncarbon surfaces, and its dependence on load, is important both in high-duty variable carbon-plate resistances and in carbon microphones. The former application was discussed by Knowles, while measurements of the electrical characteristics of microphone carbon as a function of frequency were reported by Fairweather, Frost and Shirley. Blanchard presented results on the electrical conductivity characteristics of a wide range of compressed carbon blacks, in particular in relation to their dependence on heat treatment, while Dawson and White reported on the 
properties of resistors made from various carbon blacks bonded with resins.

Methods of preparing dense, unreactive carbon are of great importance. If successful, they would find application both in bulk form and as surface coatings for less dense material. Recent work (A. R. G. Brown and W. Watt) shows that dense impervious material is produced by the thermal decomposition of hydrocarbons at a hot surface $\left(1,600-2,100^{\circ} \mathrm{C}\right.$.), and surface coatings of this kind enhance the chemical resistance of more reactive graphites. The kinetics of deposition of carbon films from benzene and toluene vapour carried by a stream of helium in a rather lower tem. perature range $\left(850-950^{\circ}\right.$ C.) have been studied by Kinney and Murphy.

Carbons as normally prepared may contain, besides small amounts of trace elements, a substantial amount of hydrogen, oxygen, nitrogen and sulphur. These elements may have an important influence on the structure, and Riley, Baraniecki and Streeter reviewed the properties of these 'heterocarbons'. The particular case of graphitic oxide has been studied extensively in the past thirty years: the present position was reviewed by de Boer and van Doorn in the light of their more recent work.

Considerable emphasis was placed on industrial carbon as a constructional material. Reviews of its applications in various industries were contributed by Lyddon and Hurden (mechanical engineering); K. F. Anderson (chemical engineering); H. D. Anderson and Ward (chemical plant) ; Hilliard (heat exchangers) ; Haines (high-temperature furnaces) ; G. E. Ross and D. W. Brown (metallurgy) ; Lakin and Mackenzie (blast-furnace linings). The mechanical properties relevant to these applications were discussed by H. W. Davidson, Losty and A. M. Ross, and by Arragon and Berthier, while methods of nondestructive testing were outlined by T. D. Davidson.

The most important new field of application of industrial graphite is in nuclear-power plant, where to the desirable properties of high density, low reactivity and mechanical strength, must be added the over-riding requirement of high purity. Discussions of this application and of the required properties were contributed by McIntosh, Heal and Cowan; Lockett; and McIntosh, O'Driscoll and Tyzack. Special attention has to be paid to the effects of high temperatures and of neutron irradiation, and papers by Hove and by Simmons were concerned with these problems. In studying the suitability of various raw materials and manufacturing procedures for nuclear-graphite production, large-scale experimental plant is needed, and that recently constructed at Harwell was described by Price and Yeats. The problems arising in the manufacture of nuclear graphite were discussed by Cornault and Rochettes. High purity may in principle be attained either by using very pure raw materials, by the purification of ordinary graphite or by purification in the course of the final graphitization. The latter method is that actually chosen, and a study of the purification of graphite by treatment with gaseous chlorine or a chlorofluorohydro. carbon during graphitization was the subject of a paper by Odening and Bowman. The resultant purity depends on the permeability of the carbon : the high density and low porosity desirable in graphite for use as a moderator may not be compatible with gas-purification methods. Methods of estimating the purity of reactor graphite were mentioned in several papers-the usual method being to compare the effect of different graphites on the time required for the reactor to become critical ; detection and estimation of rare earths by the radioactivation technique and a study of the changes of rare earth content in the course of graphite manufacture was described by Decour, Leveque, Hering and Jouquet. The alteration of properties brought about by purification was discussed by Baroin.

During the period of the Conference an exhibition of industrial carbon and graphite was held in the Department of Chemical Engineering, University College, London; and this, together with several social functions, contributed to the success of the Conference.
D. H. EveretT

\section{SAFETY IN MINES RESEARCH}

$\mathrm{T}$ HE scope of the Safety in Mines Reserrch Advisory Board was widened early in 1956 when the Board was re-appointed, with Prof. R. V. Jones as chairman, and given new terms of reference which ask the Board "to keep under review and advise the Minister of Power on the general scope, organization and progress of research work on safety in mines". Until then the scope had been restricted to the Ministry's research work on the subject. It is appropriate, therefore, for the thirty-fifth annuel report on safety in mines research (London : H.M. Stationery Office, 1958) to include a short account of the ninth international conference of directors of safety in mines research and to give in appendixes not only synopses of recent publications of the Safety in Mines Research Establishment, but also a list of papers presented by all the delegates to the ninth international conference.

The report, however, is essentially a report of the work of the Safety in Mines Research Establishment for the yeur 1956. During the year the move to new premises, in Brosd Street, Sheffield, housing the engineering sections and much of the Technical Information Service Department, was begun. It is significant that the acute problem of accommodation has resulted "as much from increasing the strength of research teams to the necessary extent, as from the increasing complexity of the work". As the director of the Establishment, Mr. H. T. Ramsay, remarks in his introduction to the report, "Mining research has been in progress for a long time and the ezsier answers have usually been found; reel advances against the remaining hazards will only be made by a penetrating study of their mechanisms and, so far from becoming easier, one may expect the Establishment's work to become more difficult".

Most of the report is concermed with the research of the Establishment into the hazards of mining. There are eight main sections, namely, explosives and blasting devices, the explosion hazard, breathing apparatus, fire hazard, engineering and metallurgical research, dust control and pneumoconiosis hazard, 